\title{
DETECTING CHANGES IN PERSISTENT SCATTERERS
}

\author{
Ramon Brcic and Nico Adam \\ Remote Sensing Technology Institute (IMF), German Aerospace Center (DLR), \\ Oberpfaffenhofen, 82234 Wessling, Germany, ramon.brcic@dlr.de
}

\begin{abstract}
In PSI (Persistent Scatterer Interferometry) the PSs are implicitly assumed to have a stable phase throughout a stack of images so that all images can be used for estimation. However, PSs can fade due to changes in acquisition geometry where the elevation or azimuth response of the PS for different baselines or zero Doppler frequencies is imprinted on the return. Physical changes caused by construction or seasonal changes due to snow cover can also cause the sudden appearance or disappearance of a PS. Here, several parametric change point estimators are assessed for their suitability in detecting such events both through simulation and their application to an ERS stack over Hamburg.
\end{abstract}

Index Terms - Change Point Estimation, InSAR, PSI, Temporal PSs

\section{INTRODUCTION}

In PSI (Persistent Scatterer Interferometry) the PSs are implicitly assumed to have a stable phase throughout a stack of images so that all images can be used for estimation. However, PSs can be expected to fade due to changes in acquisition geometry and physical changes $[1,2,3]$. A change in the zero Doppler frequency (squint angle) means that the SAR observes the PS from a different azimuth angle and so the azimuth amplitude response of the PS is imprinted on the return. Likewise, a change in perpendicular baseline (elevation angle) imprints the elevation amplitude response of the PS on the return. The degree to which acquisition geometry affects fading depends however on the physical extension of the PS and the zero Doppler or baseline spread. Fading due to physical changes can be caused by the construction and demolition of buildings with PSs suddenly appearing and disappearing or by seasonal effects such as snow cover.

At high SCR (Signal to Clutter Ratio) the phase variance is proportional to the dispersion index [4], hence changes in SCR caused by fading affect PS phase variance and PSI estimation quality. By being able to detect temporal PSs, determine where changes occur and then incorporate this information into PSI estimation, the quality can be improved. Change point estimators applied to the PS amplitudes can be used to detect such effects.

This paper analyses change point estimators for their use in the temporal PS problem. These algorithms are then applied to an ERS stack over Hamburg.

\section{CHANGE POINT ESTIMATION}

If a PS appears or disappears due to physical changes, a step function like change in PS amplitude is expected when the returns are ordered temporally. For fading due to acquisition geometry, a change in amplitude mirroring the response will be present when the amplitudes are ordered according to zero Doppler frequency or perpendicular baseline. Detecting such discontinuities is a change point estimation problem. A classical online approach is to compare samples within the first and second halves of a moving window [5]. However, in PSI we are concerned with offline or retrospective change point estimation where the data have already been collected. Both parametric and nonparametric approaches for change point estimation exist.

In the parametric case one can formulate a GLRT (Generalised Likelihood Ratio Test) [6] given the distribution of the returns. Typically the PS amplitude is modeled as Rician because of its simple physical meaning of a deterministic signal in Gaussian distributed clutter. For the not unrelated SAR image segmentation problem with a Rayleigh model, change detection based on both GLRT [7] and Bayesian [8] approaches have been proposed.

In the nonparametric case the most general formulation of the problem is to test whether two datasets follow the same distribution. A promising approach used in adaptive InSAR stack multilooking was nonparametric goodness-of-fit tests such as the KologmorovSmirnov and Anderson-Darling 2-sample tests for the equality of distributions [9]. There, the Anderson-Darling test performed well even for as few as 10 observations. Another approach is the nonparametric version of Student's t-test, the Mann-Whitney-Wilcoxon test [10], that tests whether one of two datasets has larger values than the other although it has the drawback of failing if the distributions differ significantly. This statistic was extended to the multivariate multiple change point problem in [11]. Nonparametric approaches generally require large sample sizes in order to be powerful and so they are not expected to outperform the parametric ones. For this reason they are not considered further and are left as a topic of future work.

Here we pursue parametric change point estimators for a single change based on the GLRT approach. They are fast and form the basis for more complicated multiple change point estimators that must also incorporate model selection.

\section{PARAMETRIC CHANGE POINT ESTIMATION}

The PS intensities or magnitudes are represented by a dataset $\mathcal{S}=$ $\left\{x_{n}, n=0, \ldots, N-1\right\}$ of independent samples. In the absence of a change point the $x_{n}$ are i.i.d. with PDF $f\left(x_{n} ; \theta\right)$. If a change point is present at $m_{1}$ then $\mathcal{S}$ can be split into two segments $\mathcal{S}_{1}=\left\{x_{n}: n=\right.$ $\left.0, \ldots, m_{1}-1\right\}$ and $\mathcal{S}_{2}=\left\{x_{n}: n=m_{1}, \ldots, N-1\right\}$ of length $N_{1}$ and $N_{2}$ respectively with different distributional parameters $\theta_{1} \neq$ $\theta_{2}$.

The hypothesis testing framework for detecting a change point is then

$$
\mathcal{H}_{0}: \theta=\theta_{1}=\theta_{2} \text {, no change point or one segment }
$$$$
\mathcal{H}_{1}: \theta_{1} \neq \theta_{2} \text {, one change point or two segments }
$$ 
The corresponding likelihood ratio for the GLRT is

$$
\frac{\prod_{n \in \mathcal{S}} f\left(x_{n} ; \hat{m}_{1} \mid \mathcal{H}_{1}\right)}{\prod_{n \in \mathcal{S}} f\left(x_{n} ; \hat{\theta} \mid \mathcal{H}_{0}\right)}
$$

where $\hat{m}_{1}$ is the MLE (Maximum Likelihood Estimator) of change point location obtained from maximising the likelihood function under $\mathcal{H}_{1}$

$$
\hat{m}_{1}=\arg \max _{m_{1}} \prod_{n \in \mathcal{S}_{1}} f\left(x_{n} ; \hat{\theta}_{1}\right) \prod_{n \in \mathcal{S}_{2}} f\left(x_{n} ; \hat{\theta}_{2}\right)
$$

so that $\hat{\theta}_{1}$ and $\hat{\theta}_{2}$, the MLEs of the distributional parameter(s) for segments $\mathcal{S}_{1}$ and $\mathcal{S}_{2}$ respectively, are implicitly functions of the change point. $\hat{\theta}$ is the MLE of $\theta$ estimated under $\mathcal{H}_{0}$, that is, from the entire dataset $\mathcal{S}$.

\subsection{Ratio Edge Detector}

The RED (Ratio Edge Detector) was proposed to detect changes in SAR images for the purpose of segmentation [7] and is based on Rayleigh magnitudes, hence the distributional parameter $\theta$ is the power whose MLE is the average intensity. The test uses a moving window of fixed length $2 L$ that is split into two segments of equal length $L$. For each window position the ratio of the average intensities from the two segments, $r=\hat{P}_{1} / \hat{P}_{2}$, is found and inverted if it is less than one, $r=\max (r, 1 / r)$. These power ratio values were then smoothed and used for edge detection. As noted in [7] this is a GLRT for a change in power between the two segments and can be derived using the framework outlined above with a Rayleigh model. For its purpose here, the change point location estimate is the window position that maximises the power ratio. Note that the detection rate and change point location accuracy of the RED will drop for change points located less then $L$ samples away from the edges of the time series. Reasonable results are typically obtained with window sizes of at least $2 L=20$.

\subsection{Exponential MLE}

Extending the RED to use the entire dataset instead of a fixed window length leads to the exponential (intensity) or Rayleigh (magnitude) MLE for change point location,

$$
\hat{m}_{1}=\arg \min _{m_{1}} N_{1} \ln \hat{P}_{1}+N_{2} \ln \hat{P}_{2} .
$$

This estimator will always have higher detection rates than the RED because more data are used. It can also be used to locate change points at the ends of the data whereas the RED cannot detect or will give biased estimates of the change point when it is located less than half the window length away from the ends of the data. A disadvantage is that it is slower than the RED.

\subsection{Rice MLE}

The exponential distribution describes the intensity of homogeneous patches of distributed scatterers in a SAR image assuming Gaussian returns. A simple model for the complex return of a PSs is that of a constant signal (the PS) in Gaussian noise (the clutter) so that the PS magnitude is Rice distributed. Using the Rice model in (4) leads to the Rice MLE of change point location.

MLE estimates of the Rice parameters can be obtained according to the method in [12] where it was also shown that the estimates are unique and correspond to the global maximum of the likelihood function. A safe-guarded Newton-Raphson algorithm must be used in order to ensure that the solution remains within the domain of definition. Convergence is reached in 5 iterations on average.

Unlike the other MLEs, the sufficient statistics for the Rice MLE are complicated expressions that cannot be calculated sequentially but must be calculated anew for every unique segment, leading to a substantial increase in computational complexity. This could be reduced by using the Rice parameter estimates from the current segment $\left\{x_{n}, n=0, \ldots, m_{1}-1\right\}$ to initialise the search for the next segment $\left\{x_{n}, n=0, \ldots, m_{1}\right\}$ since there is only a difference of one sample between these segments.

\subsection{Gaussian MLE}

Using the Gaussian model in (4) leads to the Gaussian MLE of change point location

$$
\hat{m}_{1}=\arg \min _{m_{1}} N_{1} \ln \widehat{\sigma_{1}^{2}}+N_{2} \ln \widehat{\sigma_{2}^{2}}
$$

where $\widehat{\sigma_{1}^{2}}$ and $\widehat{\sigma_{2}^{2}}$ are the biased sample variances of segments $\mathcal{S}_{1}$ and $\mathcal{S}_{2}$ respectively. Although the Gaussian model has no physical motivation for PSs except for being the limiting case of the Rice distribution at high SCR, it is a widely used model for change point estimation and has been heavily studied. For a fixed window length the Gaussian MLE is related to Student's t-test for equal, or Welch's test for unequal, variances. Note that the magnitudes should be used with the Gaussian MLE, not the intensities.

\subsection{Computational Complexity}

The relative computation times in IDL, normalised to the RED with a segment length of $L=10$, for $10^{6}$ PSs and 100 scenes are shown in Table 3.5. The RED requires about one minute, the Rice MLE about one day. The test was made on a fairly modest machine (Sun

\begin{tabular}{|c|c|c|c|c|}
\hline \hline & & \multicolumn{3}{|c|}{ Maximum Likelihood Estimators } \\
& RED & Exponential & Rice & Gaussian \\
\hline Time & 1.0 & 2.5 & 1200 & 3.5 \\
\hline \hline
\end{tabular}

Table 1. Relative computation times of the change point estimators for $10^{6}$ PSs and 100 scenes.

Fire V440, 4 x $1.28 \mathrm{GHz}$ single core UltraSPARC-IIIi processors). A multithreaded $\mathrm{C}++$ version on more modern machines is expected to be at least 10 times faster.

To detect two change points, the computation time for the Rice MLE is expected to increase by a factor of about $N / 6$, or 20 for 100 scenes. The exponential and Gaussian MLEs are not expected to experience such a large increase because only the first and second moments are required and this can be coded extremely efficiently using sequential methods.

For multiple change points, $M$, a brute force search grows in complexity at an exponential rate of order $O\left(N^{M}\right)$ which clearly becomes impractical very fast. This can be greatly reduced to the point where multiple change point estimation does become practical through the use of dynamic programming that optimises the search path through the solution space [6]. A requirement is that the log likelihood function under $\mathcal{H}_{1}$ can be written as the sum of the log likelihood functions of the individual segments. This requirement is fulfilled here since the intensities are assumed to be temporally independent. With dynamic programming, the computational time for more than two change points would not increase significantly. 
The detection of multiple change points due to say, seasonal effects, is then still practical for a multithreaded $\mathrm{C}++$ implementation, taking about a day for the Rice MLE and minutes for the others.

\subsection{Detection Thresholds}

The null distribution of the GLRT appears difficult to determine. Resampling techniques such as a parametric bootstrap would increase the complexity by 2-3 orders of magnitude and so are impractical. Here the thresholds were found by Monte Carlo simulation and used as a guide to determine a threshold that performs well experimentally.

Simulations show that for Rician magnitudes, the RED and exponential MLE are not CFAR (Constant False Alarm Rate) detectors. Their threshold is dependent on the SCR and so a fixed threshold cannot maintain the false alarm rate as shown in Figure 1. This is because at high SCR the average intensity varies about a very large value and so the power ratio tends to 1 . The Rice and Gaussian MLE appear to yield CFAR detectors.

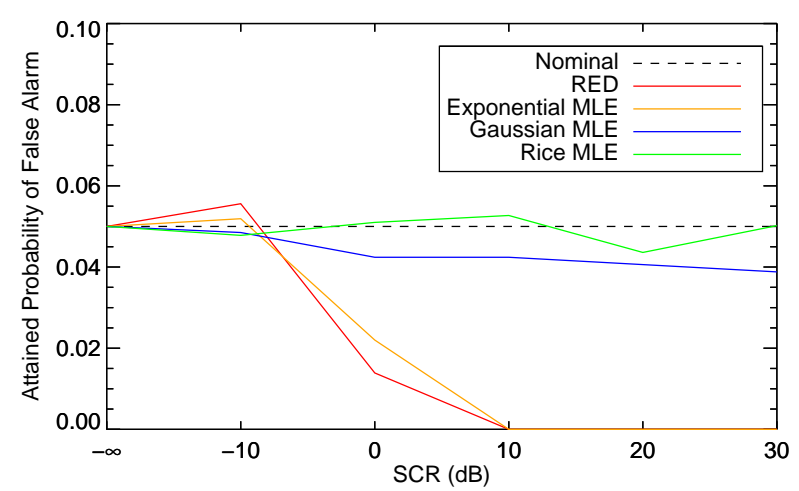

Fig. 1. Attained probability of false alarm for the 4 change point estimators under a Rician null as the SCR varies. The fixed threshold was determined for the limiting case of exponential intensities $(\mathrm{SCR}=-\infty)$

\subsection{Detection Rates}

Figure 2 shows the detection rates for the case of a PS appearing with segment 1 Rayleigh distributed and segment 2 Rice distributed, both having the same clutter power. The MLEs perform similarly and notably better than the RED, especially for change points near the ends of the time series, due to the RED using a fixed window length that does not make full use of the data.

In Figure 3 the clutter power was changed from 10 in segment 1 to 1 in segment 2 . The Rice MLE performed best, followed by the Gaussian MLE with the exponential MLE and especially the RED not performing well. In this case the expected value of the power ratio (segment 2 on segment 1$)$ was $(1+\mathrm{SCR}) / 10$, so that for SCRs near $9(9.5 \mathrm{~dB})$, the power ratio based exponential MLE and RED fail to detect the change. Hence a weakness of power ratio detectors is that they can only be relied upon if the clutter power is constant so that the problem becomes one of detecting a change in the amplitude of the PS.

Figure 4 shows the distribution of the change point estimates given the same conditions as in an Figure 2 for an SCR of $6 \mathrm{~dB}$. The

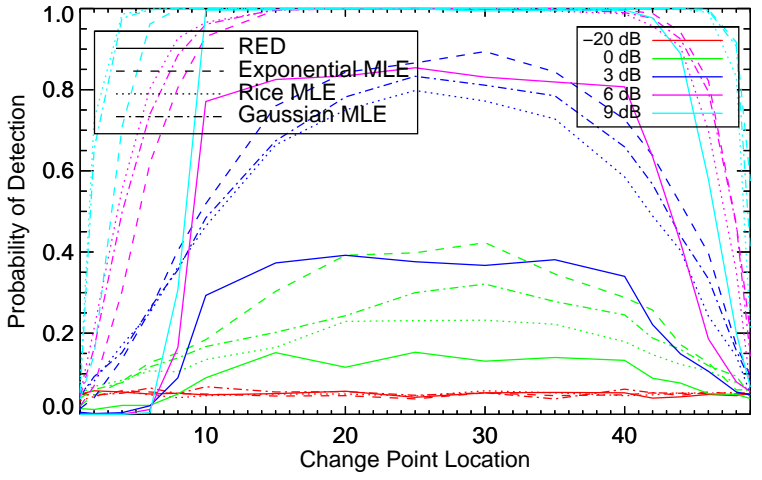

Fig. 2. Detection rates as change point location varies. Case of a PS appearing with the same clutter power before and after the change.

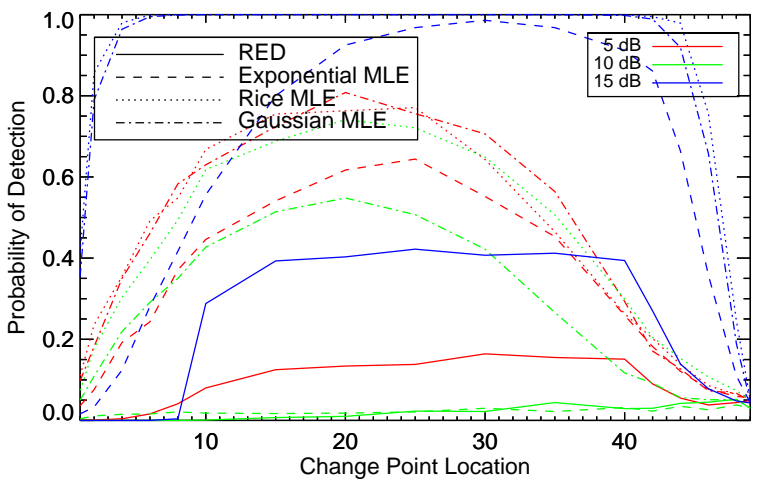

Fig. 3. Detection rates as change point location varies. Case of a PS appearing with different clutter power before and after the change.

Rice and Gaussian MLE are more tightly clustered around the true change point of 25 followed by the exponential MLE and RED.

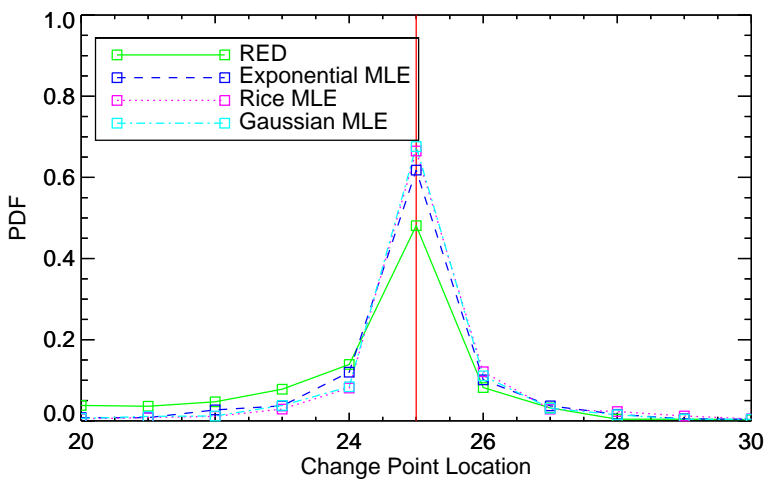

Fig. 4. Distribution of change point estimates given the same conditions as in Figure 2 for an SCR of $6 \mathrm{~dB}$. The vertical red line marks the true change point location of 25 .

In summary, the best change point estimator in view of its char- 
acteristics, performance and speed, is the Gaussian MLE.

\section{APPLICATION TO AN ERS STACK}

The 4 change point estimators were applied to a stack of 79 ERS images over Hamburg, Germany. Only temporal changes were considered as zero Doppler and baseline effects were not found.

For the 56920 PSs found in and around Hamburg, Table 4 shows the number and percentage detected as temporal PSs for a false alarm rate set to $0.01 \%$ under a Rayleigh null. Two sets of 10 PSs were then manually selected as representative of stable and temporal PSs, the corresponding false alarm and detection rates also appear in Table 4. The results do not differ significantly between estimators.

\begin{tabular}{|l|c|c|c|c|}
\hline \hline & & \multicolumn{3}{|c|}{ Maximum Likelihood Estimators } \\
& RED & Exponential & Rice & Gaussian \\
\hline No. Temporal PSs & 4063 & 8662 & 7418 & 7125 \\
Percentage & 7.1 & 15.2 & 13.0 & 12.5 \\
\hline \hline False Alarm Rate & $0 \%$ & $0 \%$ & $10 \%$ & $10 \%$ \\
Detection Rate & $90 \%$ & $100 \%$ & $100 \%$ & $100 \%$ \\
\hline \hline
\end{tabular}

Table 2. Top two rows: number and percentage of PSs detected as temporal PSs out of a total of 56920 PSs. Bottom two rows: false alarm and detection rates for two sets of 10 PSs manually selected as being stable and temporal PSs respectively.

Figure 5 shows the PS amplitude time series of a PS where a change point was detected at the same scene number 19 by all of the change point estimators. In this case the change point location appears quite clear. In other examples the change point location is not so distinct and the estimators deliver different location estimates.

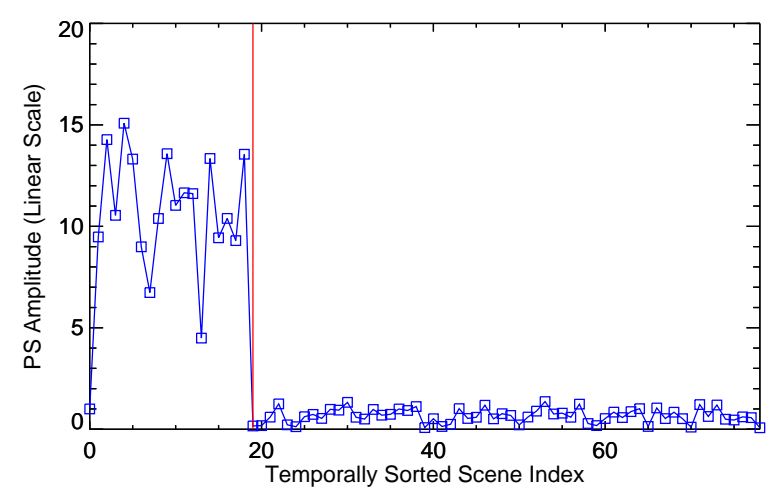

Fig. 5. Example PS amplitude time series for the Hamburg stack for a PS where a change point was detected at the same scene number 19 by all of the change point estimators.

\section{CONCLUSIONS}

We have examined several parametric change point estimators for detecting temporal PSs. The most suitable in view of its characteristics, performance and speed was the Gaussian MLE. The one disadvantage of the Rice MLE was its relative slowness while the RED and exponential MLE, although fast, had some undesirable characteristics and could not be relied upon when the clutter power was not constant. Experiments on an ERS stack showed, however, that in terms of false alarm and detection rates, all perform similarly in practice.

The extension of these detectors to the multiple change point estimation problem for detecting seasonal effects is a topic for future work. Likewise is the incorporation of information about the phase variance in different segments of a temporal PS into the PSI processing chain. It is envisaged that this could occur prior to network inversion at the estimation on arcs step where for each arc in the PS network the topography and deformation parameters are estimated using the LAMBDA method [13].

\section{REFERENCES}

[1] D. Perissin, SAR Super-Resolution and Characterization of Urban Targets, Ph.D. thesis, VDM Verlag, 2010.

[2] Alessandro Ferretti, Carlo Colesanti, Daniele Perissin, Claudio Prati, and Fabio Rocca, "Evaluating the effect of the observation time on the distribution of SAR permanent scatterers," in FRINGE 2003 Workshop, Frascati, Italy, December 2003, p. 6, ESA.

[3] Marco Basilico, Alessandro Ferretti, Fabrizio Novali, Claudio Prati, and Fabio Rocca, "Advances in permanent scatterer analysis: Semi and temporary PS," in European Conference on Synthetic Aperture Radar, Ulm, Germany, May 2004, p. 349.

[4] C. Prati A. Ferretti and F. Rocca, "Permanent scatterers in SAR interferometry," IEEE Transactions on Geoscience and Remote Sensing, vol. 39, no. 1, pp. 8-20, January 2001.

[5] M. Basseville and N. Nikiforov, The Detection of Abrupt Changes - Theory and Applications, Prentice-Hall, 1993.

[6] S. Kay, Fundamentals of Statistical Signal Processing: Detection Theory, Prentice-Hall, 1998.

[7] Ridha Touzi, Armand Lopes, and Pierre Bousquet, "A statistical and geometrical edge detector for SAR images," IEEE Transactions on Geoscience and Remote Sensing, vol. 26, no. 6, pp. 764-773, Nov. 1988.

[8] Jean-Yves Tourneret, Michel Doisy, and Marc Lavielle, "Bayesian off-line detection of multiple change-points corrupted by multiplicative noise: application to SAR image edge detection," Signal Processing, vol. 23, pp. 1871-1887, 2003.

[9] A. Parizzi and R. Brcic, "Adaptive InSAR stack multilooking exploiting amplitude statistics: A comparison between different techniques and practical results," IEEE Geoscience and Remote Sensing Letters, vol. 8, no. 3, pp. 441-445, May 2011.

[10] R. Hogg and A. Craig, Introduction to Mathematical Statistics, Pearson Education, 1995.

[11] Alexandre Lung-Yut-Fong, Céline Lévy-Leduc, and Olivier Cappé, "Robust retrospective multiple change-point estimation for multivariate data," in IEEE Statistical Signal Processing Workshop, 2011, pp. 405-408.

[12] Carlo F. M. Carobbi and Marco Cati, "The absolute maximum of the likelihood function of the Rice distribution: Existence and uniqueness," IEEE Transactions on Instrumentation and Measurement, vol. 57, no. 4, pp. 682-689, April 2008.

[13] B. Kampes, Displacement Parameter Estimation using Permanent Scatterer Interferometry, Ph.D. thesis, German Aerospace Center (DLR), 2005. 